\title{
Tracking and vertexing performance of the ATLAS Inner Detector at the LHC
}

\section{S. Martí-García*it}

IFIC-València: Centre Mixte CSIC - U. València (Estudi General), València (Spain)

E-mail: Salvador.Marti@ific.uv.es

\begin{abstract}
The ATLAS experiment at the LHC is equipped with a charged particle tracking system, the Inner Detector, composed of three subdetectors, which provide high precision measurements thanks to their fine granularity. The Pixel and the microstrip subdetectors, which use the silicon technology, are complemented with the Transition Radiation Tracker. Since the LHC startup in 2009, the ATLAS inner tracker has played a central role in many ATLAS physics analyses. Rapid improvements in the calibration and alignment of the detector allowed it to reach nearly the nominal performance in merely a few months. The tracking performance proved to be stable as the LHC luminosity increased, even for events with a large number of pile-up interactions and for the extremely dense heavy ion collisions. New developments in the offline reconstruction for the 2011 run will improve the tracking performance in high pile-up conditions.
\end{abstract}

10th International Conference on Large Scale Applications and Radiation Hardness of Semiconductor Detectors,

July 6-8, 2011

Firenze Italy

\footnotetext{
*Speaker.

On behalf of the ATLAS Collaboration
} 


\section{Introduction}

The Large Hadron Collider [1] is a particle physics accelerator built at CERN. It collides head-on bunches of protons or heavy ions. The analysis of this collisions may help to discover the Standard Model (SM) Higgs boson or new physics phenomena beyond the SM.

The ATLAS experiment [2] is a general purpose experiment that records collision events produced by the LHC. In order to fulfil its scientific objectives, ATLAS is equipped with an Inner Detector (ID) tracking system [3] embedded in a $2 \mathrm{~T}$ axial magnetic field surrounding the interaction point. The calorimetry system $[4,5]$ is located beyond the ID solenoid and combines two techniques: liquid argon and sampling technique with plastic scintillators embedded in an iron absorber. Finally a muon detector system [6] occupies the outermost region of the detector and uses an air-core toroid with strong bending power in a large volume within a light and open structure.

The LHC started its operations late 2009, by colliding proton beams at $900 \mathrm{GeV}$ center of mass energy. During 2010 and 2011 the LHC has produced proton-proton collisions at $7 \mathrm{TeV}$. The integrated luminosity recorded by ATLAS amounts to $40 \mathrm{pb}^{-1}$ in 2010 , whilst more than $1 \mathrm{fb}^{-1}$ of data were already collected during 2011 at the time of writing. LHC also collided heavy ions (lead) in 2010. Such collisions produce far more tracks [7] than the $7 \mathrm{TeV}$ proton-proton collisions [8] and they do represent a challenge for the Inner Detector tracking system of ATLAS.

This note summarizes the tracking and vertexing performance of the ATLAS Inner detector and highlights the studies on: detector alignment, material studies and particle identification.

\section{The Atlas Inner Detector}

The ATLAS Inner Detector (ID) acts as the tracking system for charged particles. It was designed to produce a robust and efficient track reconstruction. It consists of three subdetectors: two using silicon planar technology (pixels and microstrip) and a drift chamber using straw tubes filled with gas. The subdetectors are called: Pixel, SCT and TRT respectively.

All the three subsystems are divided in their turn in three parts: a barrel and two end-caps (Figure 1). The barrel part is composed of several cylindrical layers of modules. The end-caps consists of several disks or wheels of modules.
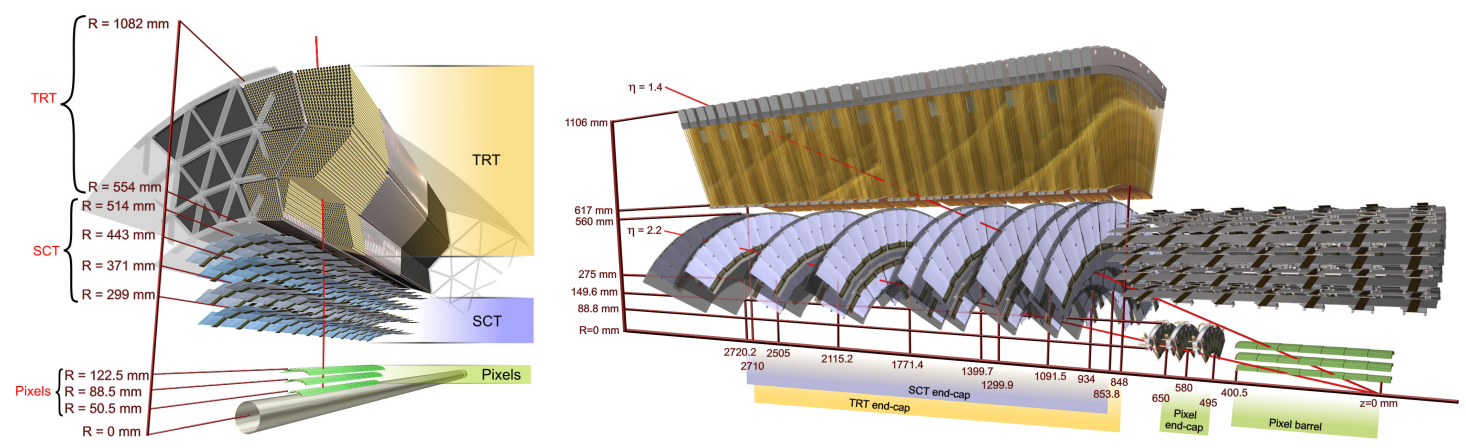

Figure 1: Schematic view of the layout of the ID barrel (left) and end-caps (right). 
There exists a superconducting solenoid which surrounds the entire tracking system and which produces a $2 \mathrm{~T}$ axial magnetic field along the beam axis direction. This magnetic field bends the trajectory of the charged particles and allows to measure their momentum.

The Pixel detector is the innermost of the ATLAS subsystems. The 1744 silicon pixel modules [9] are arranged in 3 concentric barrel layers and 3 disks in each of the end-caps. Each module has an active area of $16.4 \times 60.8 \mathrm{~mm}^{2}$ and it is segmented in pixels of $50 \times 400 \mu \mathrm{m}^{2}$. The first barrel layer is located at just $5 \mathrm{~cm}$ from the beam axis.

The silicon microstrip tracker (SCT) is built from modules assembled with two pairs of 6cm-long wafers with a pitch of $80 \mu \mathrm{m}[10,11]$. The two wafers of each side are daisy chained producing modules of strips $12 \mathrm{~cm}$ long. Each pair of sensors is glued back to back with a $40 \mathrm{mrad}$ stereo angle. There are in total 4088 SCT modules distributed in 4 concentric barrel layers and 9 disks per end-cap structure. The four wafers of each SCT modules were assembled under strict tolerances. Thanks to that, the SCT module is considered as the basic alignable unit.

The Transition Radiation Tracker (TRT) [12,13] is the outermost of the ID subdetectors (its outer radius is just above 1 meter). The detector consists of 298304 straw tubes $4 \mathrm{~mm}$ diameter. Each straw has a sensing wire in the center. The straws are arranged in 96 barrel modules (staggered in 3 rings) and 40 end-cap wheels in each end-cap. Besides the tracking capabilities, the TRT may identify electrons thanks to the transition radiation. The TRT use a gas mixture of $\mathrm{Xe}, \mathrm{CO}_{2}$ and $\mathrm{O}_{2}$.

Table 1 presents a summary of the main characteristics of the three ID subdetectors, including its nominal resolution and number of readout channels.

\begin{tabular}{lccccc}
\hline \hline Subdetector & $\begin{array}{c}\text { Radius } \\
{[\mathrm{cm}]}\end{array}$ & Element size & $\begin{array}{c}\text { Resolution } \\
\text { in precision coordinate }\end{array}$ & $\begin{array}{c}\text { Hits/track } \\
\text { in the barrel }\end{array}$ & $\begin{array}{c}\text { Readout } \\
\text { channels }\end{array}$ \\
\hline Pixel & $5-12$ & $50 \mu \mathrm{m} \times 400 \mu \mathrm{m}$ & $10 \mu \mathrm{m} \times 115 \mu \mathrm{m}$ & 3 & $80 \times 10^{6}$ \\
SCT & $30-52$ & $80 \mu \mathrm{m}$ & $17 \mu \mathrm{m}$ & 8 & $6 \times 10^{6}$ \\
TRT & $56-107$ & $4 \mathrm{~mm}$ & $130 \mu \mathrm{m}$ & 30 & $3.5 \times 10^{5}$ \\
\hline \hline
\end{tabular}

Table 1: Summary of the main characteristics of the three ATLAS ID subdetectors.

\section{Track reconstruction}

The helical trajectories of tracks are parametrised in the ATLAS software [14] as five dimensional vectors with the following parameters: $\tau=\left(d_{0}, z_{0}, \phi_{0}, \theta, q / p\right)$ where $d_{0}$ and $z_{0}$ are the transverse and longitudinal impact parameter respectively, measured with respect to the origin. $\phi_{0}$ is the azimuthal angle of the track at the point of closest approach to the origin, and $\theta$ is the polar angle. The ratio $q / p$ is the inverse of the particle momentum multiplied by its charge. The transverse momentum, $p_{\mathrm{T}}$, is computed as $p_{\mathrm{T}}=p \sin \theta$. Finally, the particle pseudorapidity $\eta$ is defined in terms of the polar angle $\theta: \eta=-\ln \tan (\theta / 2)$.

Tracks are reconstructed offline within the full ID acceptance range: $|\eta|<2.5$ using a $\chi^{2}$ fitter [15]. The pattern recognition works primarily inside-out, associating first the silicon hits and then extrapolating to the TRT to include its measurements. In a second stage, an outside-in tracking uses the TRT track segments seeds plus an inward extension in order to efficiently reconstruct 

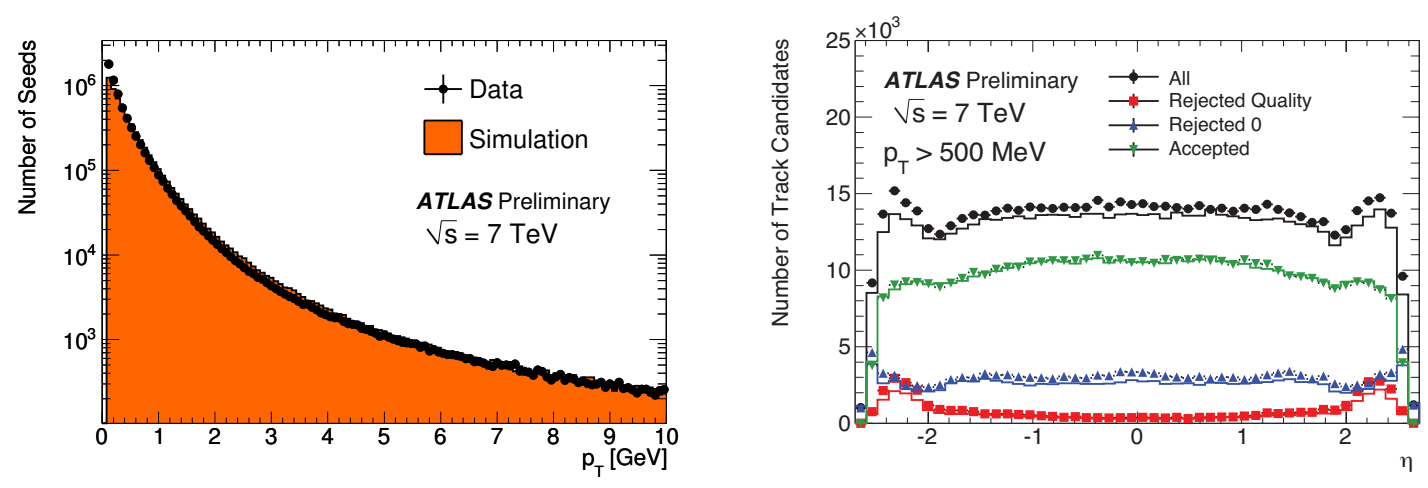

Figure 2: Left: transverse momentum distribution $\left(p_{\mathrm{T}}\right)$ of all track seeds in data compared with simulation. Right: number of track candidates as a function of $\eta$ and at different stages of the track finding. All track seeds are plotted in black and the resolved tracks in green.

tracks with secondary interactions. The pattern recognition selects first very loose track candidates and then a stringent ambiguity processor selects good track candidates (Figure 2 right). Only those satisfying a minimum number of silicon hits and a cut in the transverse and longitudinal impact parameter are kept. The tracking has been attempted as low as for $p_{\mathrm{T}}=100 \mathrm{MeV}$ (Figure 2 left).

\section{Vertex reconstruction}

Good performance of the vertex reconstruction is necessary to achieve the scientific goals of the experiment. This is specially true in case of pile-up, where many collisions occur at the same bunch crossing. The ATLAS vertex reconstruction [16] may work in an iterative or adaptive mode. In the iterative mode, first a vertex finding is executed where tracks are associated to a primary vertex and in the second stage the vertex is fitted (with a $\chi^{2}$ technique). In the adaptive vertex fitter mode (with a Kalman filter technique) the track association to the vertex is done dynamically at the time of the vertex fit, where all vertex candidates compete for the same track.

The primary vertex resolution (Figure 3 left) is extracted using a data driven method. It depends on the number of tracks as well as on the $\sqrt{\sum_{\text {tracks }} p_{\mathrm{T}}^{2}}$. For many tracks and $\sqrt{\sum_{\text {tracks }} p_{\mathrm{T}}^{2}}>8$ $\mathrm{GeV}$ the achieved resolution is $\approx 20 \mu \mathrm{m}$.

The b-tagging algorithm relies on the secondary vertex reconstruction as well as on the track impact parameter [17]. Figure 3 (right) shows the signed transverse impact parameter of the reconstructed tracks with Pixel hits. The impact parameters sign is defined with respect to the jet axis.

\section{Inner Detector Alignment}

The goal of the alignment is to provide an accurate description of the geometry of the detector: the location and orientation of every tracking element. This is vital for a proper and precise determination of the track parameters and to avoid biases. 

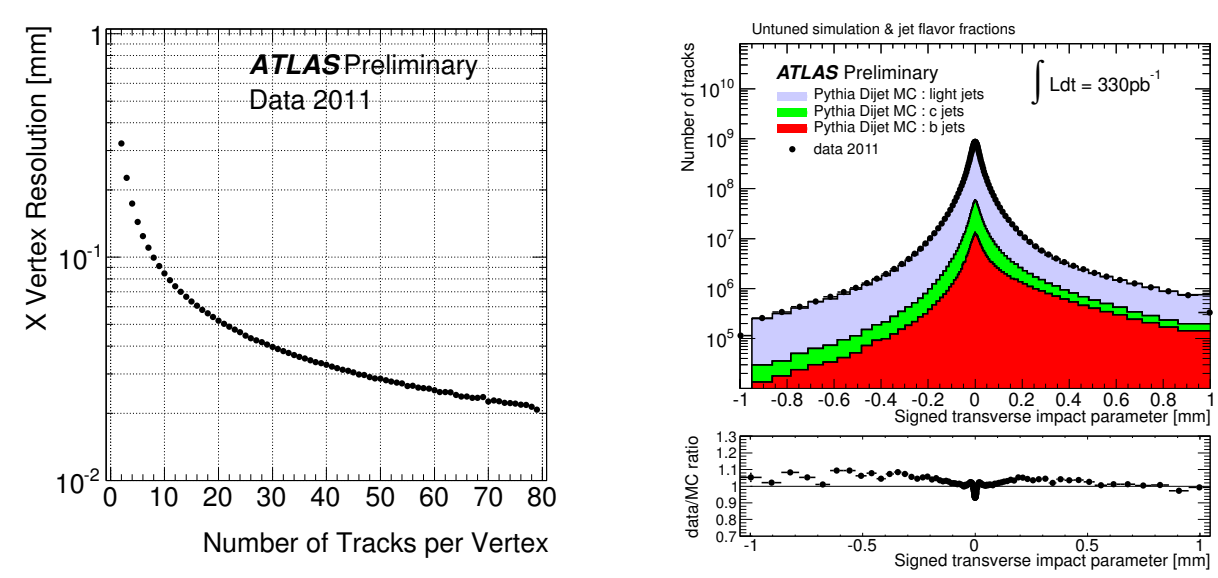

Figure 3: Left: Estimated vertex resolution using $7 \mathrm{TeV}$ data of 2011 as a function of the number of tracks per vertex. Right: signed transverse impact parameter with respect to primary vertex for tracks of b-tagging quality associated to jets, for experimental data (solid black points) and for simulated data (filled histograms for the various flavors) and the data to MC ratio.

The ID has been aligned using a track based method [18]. It consists of a $\chi^{2}$ minimization of the track-hit residuals. The algorithm is based on the minimization of the global $\chi^{2}[19,20]$ that includes a double dependence of the residuals in both alignment and track parameters.

The alignment procedure is executed at 3 different levels in accordance with the assembly of the ID structures. The level 1 corresponds to the large barrel and end-cap structures of the Pixel, SCT and TRT (just 7 structures to align). The level 2 deals with the barrel layers and end-cap disks of the Pixel and SCT (31 structures) and the barrel modules and end-cap wheels of the TRT (176 structures). Finally, the level 3 aligns each Pixel and SCT module and all TRT straw tubes. In total, one has to deal with more than 700k degrees of freedom.

There are two dedicated data streams selected by the high level trigger: a collection of high $p_{\mathrm{T}}$ and isolated collision tracks and a set of cosmic-ray tracks triggered during the empty LHC bunches. The detector stability is tested run-by-run (Figure 4 left). Alignment constants over long data taking periods are computed offline using the GRID. The alignment software allows to set constraints on the track and alignment parameters: tracks to come from the beam spot region, $p$ of muons from the Muon Spectrometer, $E / p$ for electrons and assembly survey data [21]. The reached alignment precision of the pixel barrel modules modules is $4 \mu \mathrm{m}$ and10 $\mu \mathrm{m}$ for the SCT modules. A nice data vs. MC agreement is obtained for the residuals of all parts of the ID [18].

The alignment validation comprises a detailed check of many alignment specific distributions (as the residuals of all components) as well as checks for track parameters and their errors. The resonance invariant masses (light as $K_{s}^{0}$ and heavy as $Z$, Figure 4 right) are scrutinized against all the track parameters in order to detect and correct possible biases.

\section{Material studies}

The precise knowledge of the material within the tracking volume is mandatory for an accurate track reconstruction and a precise track parameter determination. The methods to study 

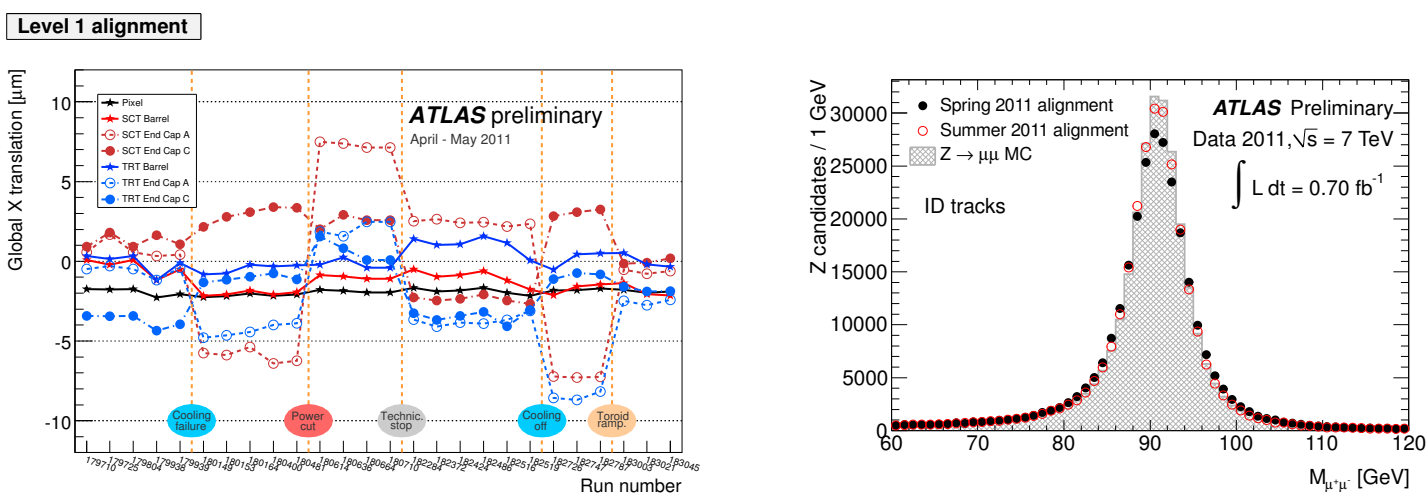

Figure 4: Left: Evolution of the alignment parameters of the seven Level 1 structures. The data spans over a period of 2 month. The detector only moves when reacting to hardware events. Otherwise, it is remarkably stable. Right: Reconstructed invariant mass of the $Z \rightarrow \mu^{+} \mu^{-}$. The improvement of the summer 2011 alignment is clearly seen. MC with a perfectly aligned detector is also included.

the amount of material and its location within the tracking volume are: photon conversions and hadronic interactions.

The comparison between real data and simulation of the spatial distribution of the reconstructed secondary vertices (either with photon conversions or hadronic interactions) helps to improve the description of material in the detector geometry. In the same sense, the rate of photon conversions inside the tracker volume compared with the beryllium beam pipe (very well known object) helps to asses the amount of material inside the real detector (see Figure 5). A good description of the supporting structures and services has been achieved [22].

\section{Particle identification}

The ID uses two particle identification techniques: energy loss $(\mathrm{dE} / \mathrm{dx})$ and transition radiation. The ionization losses of each track can be measured by the Pixel detector [23] and the probability for a particle to match a given mass hypothesis is then calculated (Figure 6 left).

The TRT provides discrimination between electrons and pions over the energy range of 1 to $200 \mathrm{GeV}$ thanks to the transition radiation technique [24]. When a particle produces transition radiation, the additional radiation makes some TRT hits go over the higher threshold (Figure 6 right).

\section{Summary and Outlook}

The ATLAS Inner Detector tracking system performance has been discussed. The Pixels, SCT and TRT are operating very efficiently and providing high quality data for the physics analysis teams. On the offline front, all the efforts dedicated to study the precise positioning of the hits recorded by the sensors, to understand the material inside the tracking volume, pattern recognition and track fitting, primary and secondary vertex fit, detector alignment and particle identification already bear fruit as the ID performance is rapidly reaching its design level. The performance of 

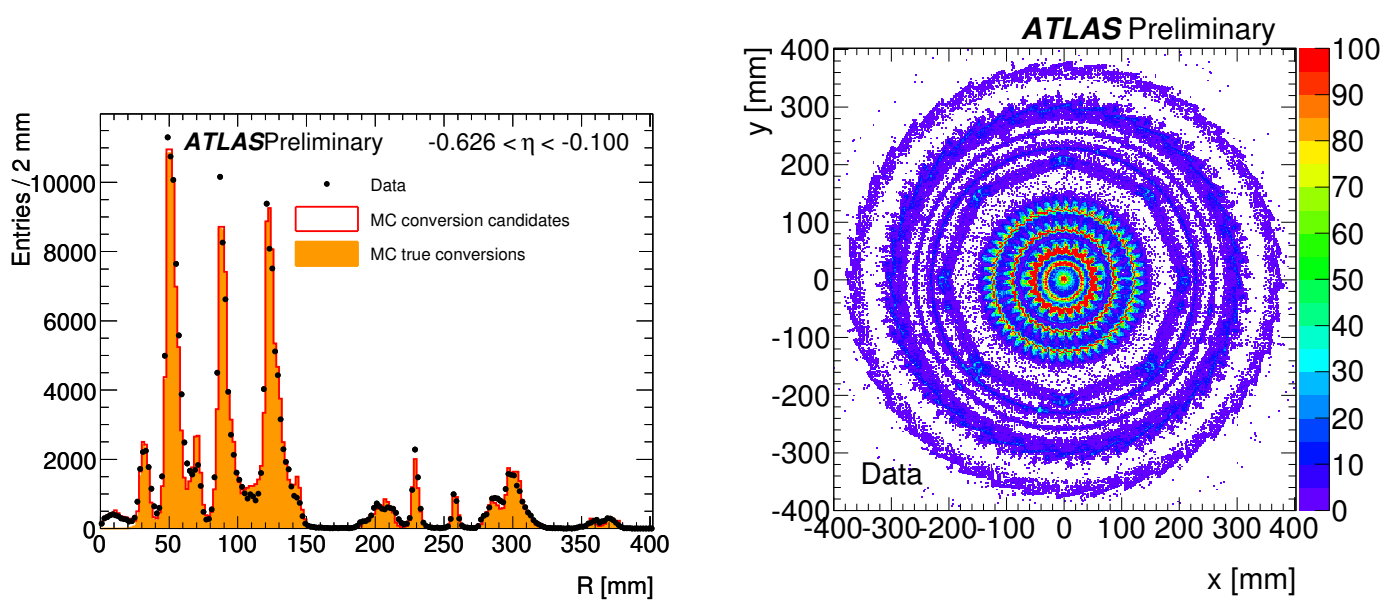

Figure 5: Left: radial distribution of the reconstructed $\gamma$ conversion vertices in the barrel tracker sections. Right: same as a function of the transverse positions (x,y). The individual Pixel and SCT modules are clearly seen as well as some supporting structures.
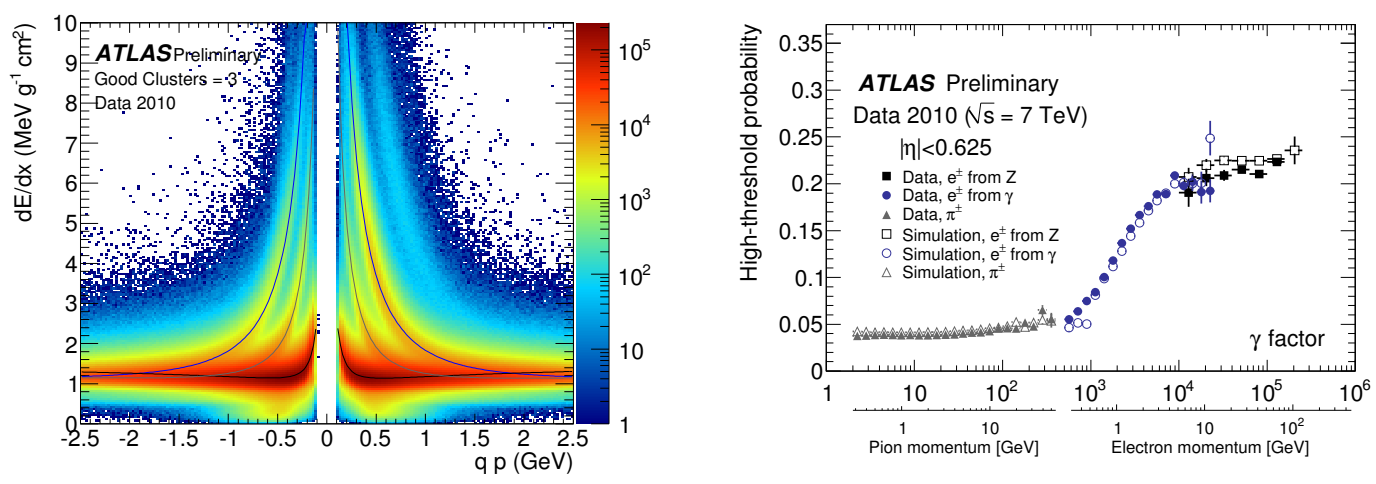

Figure 6: Left: bi-dimensional distribution of $\mathrm{dE} / \mathrm{dx}$ versus signed momentum for tracks with at least 3 good Pixel clusters. The deuteron contribution is visible in the positive side. The most probable values for protons, kaons and pions are also shown as solid black lines. Right: TRT barrel high threshold turn on curve. The value of the Lorentz $\gamma$ factor is computed assuming a mass for the candidate (electron or pion).

the ID tracking has allowed an efficient tracking during the LHC 2010 heavy ion run, which in its turn allowed to prepare the tracking for the larger interaction pile-up in LHC in 2011 in LHC.

\section{References}

[1] L. Evans and P. Bryant, LHC Machine, J. Instrum. 3 (2009) S08001.

[2] ATLAS Collaboration, The ATLAS experiment at the CERN Large Hadron Collider, J. Instrum. 3 (2009) S08003.

[3] ATLAS Collaboration, Alignment requirements, in ATLAS Inner Detector Technical Design Report, vol. I, p. 215. CERN-LHCC-1997-016, CERN, Geneva, 1997. 
[4] ATLAS Collaboration, Readiness of the ATLAS liquid argon calorimeter for LHC collisions, Eur. Phys. J. C70 (2010) 723 - 753, DOI : 10.1140 /ep jc/s10052-010-1354-y.

[5] ATLAS Collaboration, Readiness of the ATLAS Tile Calorimeter for LHC collisions, Eur. Phys. J. C70 (2010) 1193-1236, DOI : $10.1140 / \mathrm{epjc} / \mathrm{s} 10052-010-1508-y$.

[6] ATLAS Collaboration, Commissioning of the ATLAS Muon Spectrometer with cosmic rays, Eur. Phys. J. C70 (2010) 875-916, DOI : 10.1140 / ep jc / s 10052-010-1415-2.

[7] ATLAS Collaboration, G. Aad et al., Measurement of the centrality dependence of the charged particle pseudorapidity distribution in lead-lead collisions at $\left.\sqrt{(} s_{N} N\right)=2.76 \mathrm{TeV}$ with the ATLAS detector, arXiv:1108.6027 [hep-ex].

[8] ATLAS Collaboration, G. Aad et al., Charged-particle multiplicities in pp interactions measured with the ATLAS detector at the LHC, New J.Phys. 13 (2011) 053033, arXiv: 1012.5104 [hep-ex] . Long author list - awaiting processing.

[9] G. Aad et al., ATLAS pixel detector electronics and sensors, J. Instrum. 3 (2008) P09004.

[10] A. Abdesselam et al., The barrel modules of the ATLAS semiconductor tracker, Nucl. Inst. Meth. A 568 (2006) 642-671.

[11] A. Abdesselam et al., The ATLAS semiconductor tracker end-cap module, Nucl. Inst. Meth. A 575 (2007) 98-118.

[12] E. Abat et al., The ATLAS TRT Barrel Detector, J. Instrum. 3 (2008) P02014.

[13] E. Abat et al., The ATLAS TRT end-cap detectors, J. Instrum. 3 (2008) P10003.

[14] P. F. Akesson et al., ATLAS Tracking Event Data Model, ATL-SOFT-PUB-2006-004.

[15] T. Cornelissen et al., Single Track Performance of the Inner Detector New Track Reconstruction (NEWT), ATL-INDET-PUB-2008-002.

[16] ATLAS Collaboration, Performance of primary vertex reconstruction in proton-proton collisions at $\sqrt{s}=7 \mathrm{TeV}$ in the ATLAS experiment, ATLAS-CONF-2010-069.

[17] ATLAS Collaboration, Commissioning of the ATLAS high-performance b-tagging algorithms in the 7 TeV collision data, ATLAS-CONF-2011-102.

[18] ATLAS Collaboration, Alignment of the ATLAS Inner Detector Tracking System with 2010 LHC proton-proton collisions at $\sqrt{s}=7 \mathrm{TeV}$, ATLAS-CONF-2011-012.

[19] P. Bruckman, A. Hicheur and S. Haywood, Global $\chi^{2}$ approach to the alignment of the ATLAS silicon tracking detectors, ATLAS Note ATL-INDET-PUB-2005-002.

[20] A. Bocci and W. Hulsbergen, TRT alignment for SR1 cosmics and beyond, ATL-INDET-PUB-2007-009.

[21] A. Andreazza, V. Kostyukhin and R.J. Madras, Survey of the ATLAS pixel detector components, ATLAS Note ATL-INDET-PUB-2008-012.

[22] ATLAS Collaboration, G. Aad et al., A measurement of the material in the ATLAS inner detector using secondary hadronic interactions, JINST 7 (2012) P01013, arXiv:1110.6191 [hep-ex] .

[23] ATLAS Collaboration, dE/dx measurement in the ATLAS Pixel Detector and its use for particle identification, ATLAS-CONF-2011-016.

[24] ATLAS Collaboration, Particle Identification Performance of the ATLAS Transition Radiation Tracker, ATLAS-CONF-2011-128. 